ISSN: 2600-5859

\title{
La plataforma lúdica Kahoot en el aprendizaje de vocabulario en inglés
}

\section{The playful platform Kahoot in English vocabulary learning}

Daniela Fernanda Guano Merino. ${ }^{1}$, Roció de los Ángeles Barragán Murillo. ${ }^{2}$, Nancy Georgina

Rodríguez Arellano. ${ }^{3}$ \& Alexandra Marisol Terán Peralta. ${ }^{4}$

Recibido:05-01-2020 / Revisado:22-01-2020 /Aceptado: 03-02-2020/ Publicado: 05-03-2020

\begin{abstract}
DOI: https://doi.org/10.33262/concienciadigital.v3i1.2.1172

The main objective of this study was to propose the implementation of the Kahoot platform in the teaching of English vocabulary in the Level II of the Electronic Engineering in Control and Industrial Networks Career; by considering the effectiveness of the aforementioned technological tool and the main difficulties students present when learning English vocabulary. The approach was qualitative-quantitative, since both descriptive and inferential results were examined. The design was quasi-experimental, of documentary type and within the procedures four scenarios were considered; design of the measuring instruments, pretest and posttest; reliability and validity of the instruments; data collection; and results analysis and interpretation. For the sample 48 students of Level II of English were selected. During the intervention three Kahoot activities were implemented, cloze activity, quiz, and word order. From the tests applied at the beginning and at the end of the intervention, it was observed that the average of the Pre-test was 4.30 / 10 while in the Post-test the score was $7.45 / 10$. The research allows us to conclude that students were not motivated to learn vocabulary because the classes were monotonous and printed dictionaries were used, hence the importance of introducing technology in the classroom. It was also observed that the
\end{abstract}

\footnotetext{
${ }^{1}$ Escuela Superior Politécnica de Chimborazo, Facultad de Informática y Electrónica. Riobamba, Ecuador, daniela.guano@espoch.edu.ec

${ }^{2}$ Escuela Superior Politécnica de Chimborazo, Facultad de Ciencias Pecuarias. Riobamba, Ecuador, robarragan@espoch.edu.ec

${ }^{3}$ Escuela Superior Politécnica de Chimborazo, Facultad de Salud Pública. Riobamba, Ecuador, nancy.rodriguez@espoch.edu.ec

${ }^{4}$ Unidad Educativa Doce de Mayo, Área de Lengua Extranjera. Puyo, Ecuador, alexandra.teran@educacion.gob.ec
} 
activities offered by the Kahoot platform are effective, not only because there was an increase of 3.15 points in the academic performance of the students who participated in this study; but they also boosted the level of creativity and collaborative work of the students. Keywords: Technology, Vocabulary, Creativity, Collaborative Work.

\section{Resumen}

El objetivo principal del presente estudio fue proponer la implementación de la plataforma Kahoot en la enseñanza de vocabulario en inglés en el Nivel II de la Carrera de Ingeniería Electrónica en Control y Redes Industriales, considerando la efectividad de la mencionada herramienta tecnológica y las principales dificultades que presentan los estudiantes al momento de aprender vocabulario en inglés. El enfoque que se utilizó es cuali-cuantitativo, pues se examinaron resultados tanto descriptivos como inferenciales. El diseño fue cuasiexperimental, de tipo documental y dentro del procedimientos se consideraron cuatro escenarios: diseño de los instrumentos de medición, Pretest y Post-test; confiabilidad y validez de los instrumentos; recolección de datos e información; y análisis e interpretación de resultados. Para la muestra se seleccionaron 48 estudiantes del Nivel II de inglés. Durante la intervención se implementaron tres actividades de la plataforma Kahoot: cloze activity, quiz, y word order. Como resultado de los datos de los exámenes aplicados al inicio y al final de la intervención se observa que el promedio del Pretest fue de 4,30/10 mientras que el del Post-test correspondió a 7,45/10. La investigación permite concluir que los estudiantes no se sentían motivados a aprender vocabulario debido a que las clases eran monótonas y se utilizaban diccionarios impresos, de ahí la importancia de introducir tecnología en las aulas. También se pudo observar que las actividades que ofrece la plataforma Kahoot son efectivas, no solamente porque existió un incremento de 3,15 puntos en el rendimiento académico de los estudiantes que participaron en el estudio; sino que también se potenció el nivel de creatividad y trabajo colaborativo de los estudiantes.

Palabras claves: Tecnología, Vocabulario, Creatividad, Trabajo Colaborativo.

\section{Introducción}

La enseñanza-aprendizaje de inglés en el sistema educativo ecuatoriano se ha convertido en una prioridad; puesto que abre nuevas puertas culturales y sociales, a través de mayores 
oportunidades de estudio y trabajo, logrando así la realización integral profesional y personal de los individuos (Heraldo \& Jansson, 2011).

Es así que, la enseñanza de inglés ha introducido nuevas herramientas, las mismas que ofrecen ventajas como: la autonomía del usuario, la personalización del proceso de aprendizaje, la retroalimentación inmediata de los resultados de una actividad; potenciando así la exposición al idioma, que es una de las claves para aprender inglés (Villasante, 2017).

Estas herramientas se denominan Tecnologías de la Información y la Comunicación; y están presentes en nuestras vidas diarias. En el ámbito educativo, el uso de las nuevas tecnologías ha evolucionado el aprendizaje; puesto que ahora los estudiantes tienen una nueva disposición a la hora de aprender y es responsabilidad de los docentes cubrir sus necesidades (Romero, 2016).

La implementación de las nuevas tecnologías en el aula de inglés busca motivar, incentivar y atraer la atención de los estudiantes con el apoyo de dispositivos móviles y generar oportunidades de repasar, recordar y poder en práctica lo aprendido de manera lúdica; considerando que los alumnos del siglo XXI demandan nuevas experiencias (Martínez, 2017).

La plataforma Kahoot combina el aprendizaje, las nuevas tecnologías y el juego, logrando que los estudiantes se diviertan a través de actividades como debates, encuestas y cuestionarios. Es un recurso interactivo, dinámico y sencillo pues únicamente requiere de conexión Wifi y un dispositivo móvil (Martínez, 2017).

Por lo anteriormente expuesto, el objetivo del presente trabajo es analizar la efectividad de la plataforma Kahoot en el aprendizaje de vocabulario en inglés; para lo cual se citan tres investigaciones previas relacionadas sobre el tema planteado.

La primera investigación titulada "La aplicación Kahoot para mejor el aprendizaje de vocabulario en los estudiantes de colegio" plantea la implementación de Kahoot en las clases de inglés de estudiantes de Noveno Año de Educación Básica enfocada a la habilidad de presentarse.

Una vez finalizada la intervención, el autor concluye que los resultados de aprendizaje de los estudiantes mejoró; como lo muestran los promedios de las pruebas aplicadas al inicio y al final de la intervención, los mismos que fueron de 59, 23 y 84, 58 respectivamente; probando así no solamente la efectividad de la plataforma Kahoot en el aprendizaje de vocabulario, sino también que los estudiantes se sintieron motivados y disfrutaron de las actividades basadas en juegos, y tuvieron la oportunidad de trabajar de manera colaborativa mientras realizaban trabajos grupales 
ISSN: 2600-5859

(Mansur \& Fadhilawati, 2019).

En el estudio publicado en la revista Journal of English Language Studies, Susilo (2019), manifiesta que las actividades basadas en aplicaciones en línea, como Kahoot motivan el aprendizaje de inglés, logrando que los estudiantes mejoren su rendimiento de manera significativa y comprendan el valor de la implementación de Tecnologías de la Información y la Comunicación en el aula de inglés. También recomienda que los docentes saquen el mayor provecho de las nuevas tecnologías y planifiquen sus actividades cuidadosamente para que puedan promover un aprendizaje significativo y de calidad (Susilo, 2019).

La tercera investigación que lleva como título "El uso de Kahoot en el aula de inglés como Lengua Extranjera," el autor señala que los juegos como Kahoot son una excelente opción para enseñar a los estudiantes en el ambiente escolar; ya que, los estudiantes se sienten motivados a usar dispositivos móviles como celulares o tabletas, introduciendo de esta manera la tecnología en el aula; las mismas que propician un ambiente positivo, donde los estudiantes aprenden jugando.

Adicionalmente esta herramienta fomenta la competencia amistosa y el aprendizaje cooperativo (Montaner, 2018).

\section{Revisión Literaria}

\section{La importancia del vocabulario en una lengua extranjera}

El vocabulario es fundamental en la adquisición de un idioma extranjero; sin embargo, el proceso de enseñanza del mismo se ha reducido al uso de una lista de palabras que deben ser memorizadas por el estudiante de acuerdo al tema que se va a desarrollar durante una lección; lo que deja claro que existe la necesidad de incluir metodologías que hagan posible un aprendizaje significativo.

La clave para la enseñanza eficaz de vocabulario es que el estudiante aprenda no sólo el léxico de una palabra aislada, sino los múltiples elementos que la componen. El estudiante debe ser capaz de utilizar varias combinaciones de elementos léxicos que le permitan comunicar sus ideas con mayor precisión; es así, que cómo enseñar vocabulario en clase se convierte en un desafío para los profesores de inglés.

Por tanto, se debe tomar en consideración que la motivación juega un papel preponderante en el aprendizaje de inglés. Los docentes deben incorporar a sus clases actividades interesantes que incluyan el uso de las nuevas tecnologías y que promuevan el aprendizaje de vocabulario de 
manera gradual; logrando que los estudiantes desarrollen la habilidad de reconocer una palabra antes de usarla e integrarla a su léxico. En este proceso se distinguen cuatro etapas: 1) el estudiante identifica la nueva palabra; 2) aprende a reconocerla con la ayuda del docente; 3) el estudiante reconoce la palabra como propia; y 4) reconoce y produce su propio vocabulario (Muñoz, 2014).

\section{Kahoot}

Kahoot, es una plataforma de aprendizaje mixto basado en el juego, que permite a los docentes y a los estudiantes investigar, crear, colaborar y compartir conocimientos; además motiva la participación activa de los estudiantes dentro del aula, desarrollando su creatividad en un ambiente colaborativo (Romero, 2016).

La plataforma Kahoot permite crear un divertido juego de aprendizaje en cuestión de minutos y promueve la discusión y el impacto pedagógico; y lo que es más importante los estudiantes también pueden crear sus propios Kahoots para profundizar en los temas presentados, participar en discusiones dirigidas por sus compañeros.

Kahoot emula al programa de TV ¿Quién quiere ser millonario? pero la diferencia es que en esta plataforma pueden participar hasta 100 concursantes; básicamente el docente crea las preguntas sobre cualquier tema o en cualquier idioma, y los estudiantes responden las preguntas en tiempo real desde cualquier dispositivo, ya sea celular, tableta o computadora; de esta manera, el aprendizaje se activa, despertando y aumentando la predisposición por aprender. Lo que hace de Kahoot una plataforma muy efectiva es que no se limita a una pedagogía unidireccional, en la que el docente crea preguntas y los estudiantes contestan; sino que puede cambiar el rol y hacer los estudiantes jueguen entre ellos, fortaleciendo la curiosidad, la indagación y el buen hábito de formular preguntas (Gallegos, 2015).

\section{Utilizando Kahoot en el aula de inglés}

Las nuevas herramientas de aprendizaje que generalmente se combinan con la tecnología, permiten que las clases de inglés sean más dinámicas e innovadoras. La herramienta Kahoot específicamente permite que el estudiante consolide su aprendizaje de vocabulario. La ventaja de implementar este tipo de herramientas en el aula de inglés es que favorece el aprendizaje a través del juego (Espeso, 2019).

Son muchas las actividades que se pueden realizar en la clase para mantener la atención y enseñar inglés de forma más fácil; sin embargo, este estudio propone tres: 
- $\quad$ Actividades de opción múltiple

- Actividades de ordenar

- $\quad$ Actividades de encuesta

|Las actividades de opción múltiple son aquellas que se pueden introducir en una secuencia para completar o como una pregunta para ser contestada; para lo cual también se pueden usar imágenes como guía para identificar palabras que puede ser sobre un tópico específico abordado en clase con anterioridad.

Las actividades de ordenar se introducen mediante una secuencia de números, palabras, o dibujos que son ordenados. Para enfatizar el uso de vocabulario, el docente puede presentar un ejercicio donde el estudiante debe ordenar palabras de acuerdo a su categoría; por ejemplo, ocupaciones, miembros de la familia, etc.

Las actividades de encuesta se dirigen a conocer la opinión de los estudiantes. Una vez terminada la lección o la unidad, el docente puede crear una encuesta de satisfacción donde los estudiantes puedan manifestar sus opiniones en cuanto a las ventajas o quizá desventajas sobre el uso de la plataforma Kahoot dentro del aula de inglés; lo que a la vez permitirá al docente hacer una evaluación y mejorar el proceso de enseñanza aprendizaje de inglés con el apoyo de herramientas tecnológicas.

Algunas de las técnicas más efectivas para enseñar vocabulario a través del uso de Kahoot, se detallan a continuación:

\section{Close activity}

Este tipo de actividades en las que los estudiantes deben llenar espacios en blanco sirven para afianzar los conocimientos de vocabulario y reparar lo estudiado. Se puede crear una secuencia y dejar puntos en blanco en los cuales se puedan escribir diversos elementos como verbos en sus diferentes formas, verbos modales o partículas interrogativas. En el gráfico 1 se muestra un ejemplo. 
ISSN: 2600-5859

Vol. 3, $\mathrm{N}^{\circ} 1$, p. 44-62, marzo, 2020

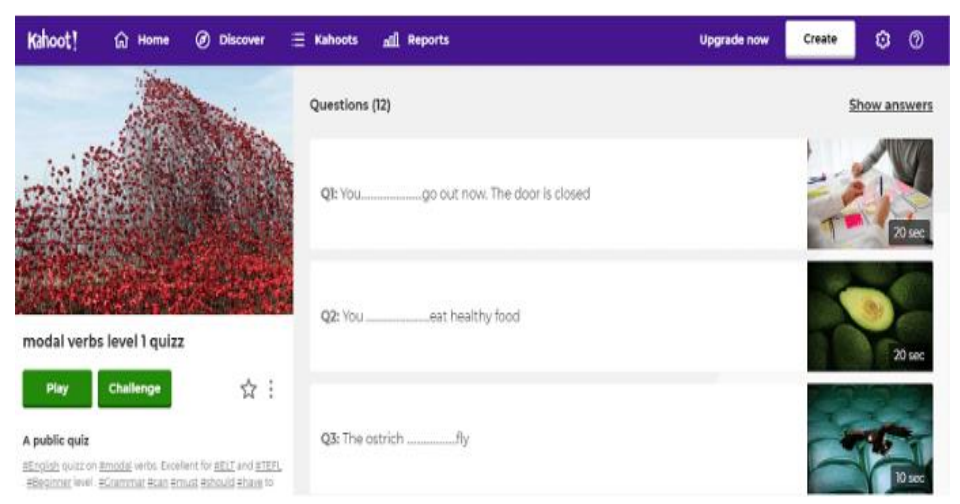

Figura 1. Cloze Activity

Fuente: Internet Autor: Educación 3.0

\section{Quiz}

Las pruebas se presentan como una alternativa eficaz al momento de aprender o repasar vocabulario; en esta actividad el estudiante debe completar la palabra que falta.

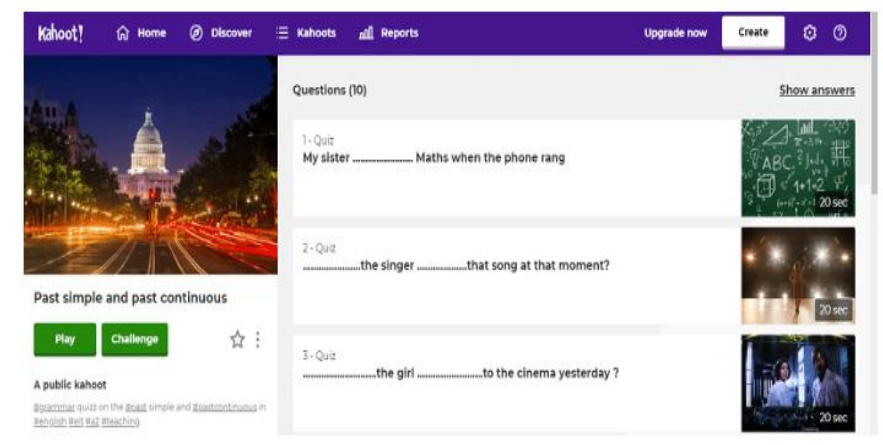

Figura 2. Quiz

Fuente: Internet Autor: Educación 3.0

\section{Questions}

Con la finalidad de aprender o repasar vocabulario se pueden crear los siguientes tipos de preguntas:

- $\quad$ Preguntas en las que se le pide al estudiante traducir de inglés a español o viceversa de acuerdo al objetivo planteado en la lección.

Preguntas relacionadas con las imágenes que se presentan, en relación al contenido de 
ISSN: 2600-5859

vocabulario.

\section{Word Order}

Esta actividad es muy práctica para enseñar inglés con Kahoot; pues presenta una estructura determinada y un orden de elementos fijo en la oración. Utilizando esta herramienta, los estudiantes podrán:

- Ordenar los distintos elementos de una pregunta.

- Ordenar adjetivos en una oración.

- Ordenar adverbios (frequency and mid-sentence adverbs)

- Ordenar distintos verbos auxiliares en inglés (to be, have got, verbos modales).

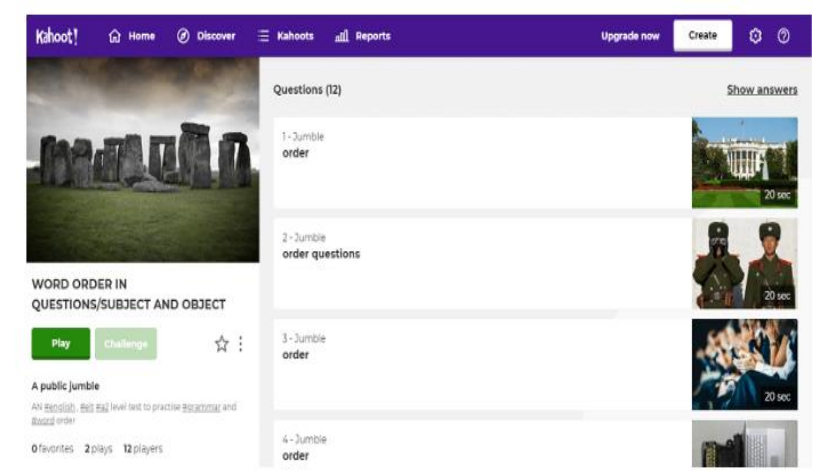

Figura 3. Word order

Fuente: Internet Autor: Educación 3.0

Finalmente, el objetivo es que los estudiantes memoricen palabras y su orden de manera intuitiva y que las actividades encaminadas a aprender vocabulario sean dinámicas y promuevan el desarrollo de la creatividad y trabajo colaborativo dentro del aula de inglés como lengua extranjera (Espeso, 2019).

\section{Objetivos}

\section{Objetivo General}

- Proponer la implementación de la plataforma Kahoot en la enseñanza de vocabulario en inglés en el Nivel II de la Carrera de Ingeniería Electrónica en Control y Redes Industriales. 
ISSN: 2600-5859

\section{Objetivos Específicos}

- Identificar las principales causas del bajo nivel de vocabulario de los estudiantes de la Carrera de Ingeniería Electrónica en Control y Redes Industriales.

- Explicar las características y beneficios de la plataforma lúdica Kahoot en el aprendizaje de vocabulario en inglés.

- Mencionar 3 actividades que motiven el aprendizaje de vocabulario en inglés con el uso de la plataforma Kahoot.

- Determinar la efectividad de la implementación de la plataforma lúdica Kahoot en el incremento del vocabulario en inglés en los estudiantes del Nivel II de la Carrera de Ingeniería Electrónica en Control y Redes Industriales.

\section{Metodología}

Esta investigación es cuali-cuantitativa debido a que los datos fueron extraídos y comparados de la aplicación del Pre y Post test dirigido a 48 estudiantes del Nivel II de inglés paralelo "A" y "B" de la Carrera de Ingeniería Electrónica en Control y Redes Industriales de la Escuela Superior Politécnica de Chimborazo en la cuidad de Riobamba.

El diseño te investigación fue de tipo documental ya que permitió analizar la información escrita en diversos medios impresos y digitales sobre el tema de estudio, los mismos que permitieron apoyar, analizar y obtener claras conclusiones que apoyen al tema objeto de estudio de esta investigación.

Como dice su nombre la investigación documental es el método basado en la revisión de textos, artículos, bibliografías, videos, películas entre otros ya existentes sobre un tema y que pueden ser utilizadas para dar inicio o traer a flote un tema ya tratado. Allí se puede encontrar una investigación histórica hecha ya sobre el tema de interés. (Restrepo, 2016, pág. 5)

El diseño de investigación también fue cuasi - experimental debido a que los investigadores manipularon las variables de estudio para poder medir el efecto de cada una de ellas y al mismo tiempo ayudaron a medir el grado de relación entre las variables de la población estudiada.

Por los objetivos de la investigación, fue un estudio de tipo descriptivo porque se conocía de la población de 48 estudiantes del Nivel II de inglés paralelo "A" y "B" de la Carrera de Ingeniería Electrónica en Control y Redes Industriales; mismos que fueron objeto de estudio para poder llevar a cabo este trabajo investigativo y a la vez buscar las propiedades y características 
importantes de los objetivos propuestos, así también se consideró el lugar de los hechos y el tiempo que se dispuso para recolectar toda la información necesaria para el desarrollo de esta investigación.

La investigación descriptiva exhibe el conocimiento de la realidad tal como se presenta en una situación de espacio y de tiempo dado. Aquí se observa y se registra, o se pregunta y se registra. Describe el fenómeno sin introducir modificaciones: tal cual. Las preguntas de rigor son: ¿Qué es?, ¿Cómo es?, ¿Dónde está?, ¿Cuándo ocurre?, ¿Cuantos individuos o casos se observan?, ¿Cuáles se observan? (Rojas, 2015, pág. 7)

Los instrumentos de la investigación se construyeron a través de los siguientes procedimientos que se siguieron:

- Diseño del instrumento de evaluación Pre Test y Post Test (40 preguntas de vocabulario)

- Confiabilidad y Validez de los instrumentos a evaluación. (Validado por docentes y director de carrera)

- Procedimiento para la recolección de datos e información. (Evaluación de Pre y Post Test) - Análisis e interpretación de la información. (Recopilación y tabulación de los datos obtenidos)

Estos procesos permitieron descubrir, analizar, discutir e interpretar la información que se encuentra concentrada tanto en las tablas de datos como en los gráficos estadísticos desplegados en este trabajo investigativo. Donde el análisis de los resultados se realizó en función de los objetivos específicos. (Universidad Central del Ecuador, 2015, pág. 5)

El aprendizaje de vocabulario es muy importante por la necesidad de aprender un nuevo idioma y desarrollar las habilidades básicas del idioma. La constante dinámica para la adquisición de nuevo vocabulario ha conllevado a utilizar diversas técnicas y estrategias pero que se han convertido monótonas. Es así que a través de este trabajo de investigación se aplicó el uso de la plataforma Kahoot en miras a la adquisición activa y progresiva de nuevo vocabulario para el estudio del idioma inglés como lengua extranjera con los 48 estudiantes del Nivel II de inglés paralelo "A" y "B" de la Carrera de Ingeniería Electrónica en Control y Redes Industriales, donde los estudiantes participaron de forma constante, y activa aplicando sus conocimientos de vocabulario junto al uso de la tecnología de acuerdo a las unidades estudiadas durante el semestre académico.

Kahoot, es una plataforma de aprendizaje y trivial basada en juegos. ¡Qué hace Kahoot! 
Tan genial es que tiene usos más allá del aula; se puede usar en oficinas y en entornos sociales, por lo que es un éxito para todas las edades. Kahoot se puede utilizar desde cualquier dispositivo (a través del sitio web o la aplicación) haciendo el aprendizaje divertido e incluso en todos los contextos. Loa maestros pueden crear juegos de opción múltiple relacionados con el contenido de la clase que pueden hacer el aprendizaje divertido. (Mendoza, 2018)

\section{Resultados}

Los resultados de los análisis obtenidos en la investigación mostraron la mejora que consiguieron los 48 estudiantes del Nivel II de inglés paralelo " $\mathrm{A}$ ” y “ $\mathrm{B}$ ” de la Carrera de Ingeniería Electrónica en Control y Redes Industriales en el aprendizaje de nuevo vocabulario en ingles a través de la plataforma Kahoot.

Como se indicó anteriormente el proceso de investigación se lo realizó durante los tres periodos parciales de estudio que tiene el semestre académico.

Al inicio del primer periodo se identificó a través de un Pre test (Tabla 1) de 40 preguntas el bajo nivel de vocabulario que poseían los estudiantes debido a que fracasaban en sus tareas asignadas y en la participación activa en clases por la falta de vocabulario al momento de realizar sus actividades y esto se evidencio cuantitativamente. Ya que se estaba dando un proceso monótono y tradicional para aprender vocabulario como fue a través de diccionarios impresos y tarjetas con imágenes en las clases de inglés.

Posteriormente, después de cada unidad de estudio se propone y se aplica la plataforma Kahoot con actividades de vocabulario a fin de obtener la participación activa, dinámica y continua de los estudiantes en forma individual y en parejas. Es así que, ya para el tercer periodo de estudios se propusieron y establecieron tres actividades que motivaron el aprendizaje en forma significativa de vocabulario de inglés con el uso de la plataforma Kahoot: actividades de opción múltiple, actividades de ordenar y actividades de encuesta, todas estas actividades permitieron mantener la atención y enseñar vocabulario en inglés.

Finalmente, se aplicó un Post Test (Tabla 2) donde se pudo constatar el nivel superior de vocabulario adquirido por los 48 estudiantes del Nivel II de inglés paralelo "A" y "B" de la Carrera de Ingeniería Electrónica en Control y Redes Industriales. 
ISSN: 2600-5859

Vol. 3, N¹, p. 44-62, marzo, 2020

Tabla 1

Resultados Individuales Pre Test.

ESCUELA SUPERIOR POLITÉCNICA DE CHIMBORAZO

FACULTAD DE INFORMÁTICA Y ELECTRÓNICA - INGLES NIVEL II ESTUDIANTES NOTAS PRE TEST

PARALELO "A" PARALELO "B"

\begin{tabular}{lll}
\hline $\mathbf{1}$ & 5,7 & 3,6 \\
$\mathbf{2}$ & 6,9 & 2,4 \\
$\mathbf{3}$ & 2,2 & 5,4 \\
$\mathbf{4}$ & 4,5 & 4,2 \\
$\mathbf{5}$ & 3,9 & 3,3 \\
$\mathbf{6}$ & 3,0 & 3,0 \\
$\mathbf{7}$ & 5,1 & 3,6 \\
$\mathbf{8}$ & 6,0 & 5,4 \\
$\mathbf{9}$ & 2,7 & 3,9 \\
$\mathbf{1 0}$ & 8,5 & 3,0 \\
$\mathbf{1 1}$ & 2,4 & 3,6 \\
$\mathbf{1 2}$ & 3,2 & 5,1 \\
$\mathbf{1 3}$ & 3,0 & 3,0 \\
$\mathbf{1 4}$ & 2,7 & 2,7 \\
$\mathbf{1 5}$ & 4,8 & 3,6 \\
$\mathbf{1 6}$ & 6,9 & 2,4 \\
$\mathbf{1 7}$ & 7,8 & 6,3 \\
$\mathbf{1 8}$ & 7,5 & 2,7 \\
$\mathbf{1 9}$ & 6,3 & 3,3 \\
$\mathbf{2 0}$ & 3,3 & 6,6 \\
$\mathbf{2 1}$ & & 3,3 \\
$\mathbf{2 2}$ & & 3,6 \\
$\mathbf{2 3}$ & & 5,4 \\
$\mathbf{2 4}$ & & 2,4 \\
$\mathbf{2 5}$ & & 2,1 \\
$\mathbf{2 6}$ & & 5,4 \\
$\mathbf{2 8}$ & & 3,3 \\
PROMEDIO & & 4,5 \\
\hline & & $\mathbf{3 , 8}$ \\
\hline
\end{tabular}

Fuente: Notas Pre Test aplicado estudiantes Nivel II paralelo A y B

Autor: Investigadores

Como indican los datos en la Tabla 1, debemos puntualizar que los 48 estudiantes del Nivel II de inglés paralelo "A" y "B" de la Carrera de Ingeniería Electrónica en Control y Redes Industriales; reciben una valoración sobre 10 puntos luego de haber realizado el Pre Test, lo cual nos permite ver su nivel de vocabulario real al momento de iniciar el semestre académico. Este pre test permitió notar que la mayoría de estudiantes tiene un nivel muy bajo en torno al conocimiento de vocabulario lo cual impedía seguir el proceso de enseñanza aprendizaje del idioma inglés al saber que es un factor indispensable para la adquisición de un nuevo idioma. De tal manera que luego de haber hecho el análisis del grupo de estudiantes del Nivel II de inglés paralelo "A" y "B" 
ISSN: 2600-5859

como promedio general tienen 4,8 sobre 10 puntos paralelo " $\mathrm{A}$ " y un promedio general de 3,8 paralelo "B".

No obstante, luego de la aplicación de la plataforma Kahoot con diferentes actividades de aprendizaje interactivo y secuencial con los estudiantes durante los tres periodos de estudio; se aplicó el Post Test con la misma población que fue motivo de esta investigación obteniendo resultados positivos en las notas individuales como se muestra en la Tabla 2, pero también en el promedio general alcanzado por los estudiantes pertenecientes al Nivel II de inglés paralelo " $\mathrm{A}$ " $\mathrm{y}$ "B" como se puede evidenciar por medio de las notas individuales y promedios generales que se aprecian en las tablas 1 y 2 .

\section{Tabla 2}

Resultados Individuales Post Test

ESCUELA SUPERIOR POLITÉCNICA DE CHIMBORAZO FACULTAD DE INFORMÁTICA Y ELECTRÓNICA - INGLES NIVEL II ESTUDIANTES NOTAS POST TEST PARALELO "A" PARALELO "B"

\begin{tabular}{lll}
\hline $\mathbf{1}$ & 8,7 & 6,6 \\
$\mathbf{2}$ & 9,6 & 6,6 \\
$\mathbf{3}$ & 4,2 & 9,9 \\
$\mathbf{4}$ & 9,8 & 7,2 \\
$\mathbf{5}$ & 8,4 & 6,0 \\
$\mathbf{6}$ & 7,5 & 8,6 \\
$\mathbf{7}$ & 6,6 & 8,1 \\
$\mathbf{8}$ & 9,9 & 8,6 \\
$\mathbf{9}$ & 8,4 & 8,7 \\
$\mathbf{1 0}$ & 9,5 & 6,3 \\
$\mathbf{1 1}$ & 3,9 & 3,0 \\
$\mathbf{1 2}$ & 4,2 & 6,6 \\
$\mathbf{1 3}$ & 8,4 & 5,1 \\
$\mathbf{1 4}$ & 5,7 & 7,5 \\
$\mathbf{1 5}$ & 7,5 & 6,3 \\
$\mathbf{1 6}$ & 9,3 & 6,0 \\
$\mathbf{1 7}$ & 8,9 & 9,9 \\
$\mathbf{1 8}$ & 9,3 & 9,0 \\
$\mathbf{1 9}$ & 7,5 & 8,1 \\
$\mathbf{2 0}$ & 6,6 & 8,6 \\
$\mathbf{2 1}$ & & 6,0 \\
$\mathbf{2 2}$ & & 7,5 \\
$\mathbf{2 3}$ & & 5,4 \\
$\mathbf{2 4}$ & & 5,7 \\
$\mathbf{2 5}$ & & 9,5 \\
$\mathbf{2 6}$ & & 8,1 \\
$\mathbf{2 7}$ & & 6,0 \\
$\mathbf{2 8}$ & $\mathbf{7 , 7}$ & 7,5 \\
PROMEDIO & & $\mathbf{7 , 2}$ \\
\hline Fut & & \\
\hline
\end{tabular}

Fuente: Notas Post Test aplicado estudiantes Nivel II paralelo A y B Autor: Investigadores 
ISSN: 2600-5859

Al final de la investigación se pudo apreciar el nivel de adquisición de vocabulario significativo por parte de los estudiantes del Nivel II de inglés paralelo "A" $\mathrm{y}$ "B" a través de la plataforma Kahoot como herramienta en la enseñanza aprendizaje de nuevas palabras en el idioma inglés.

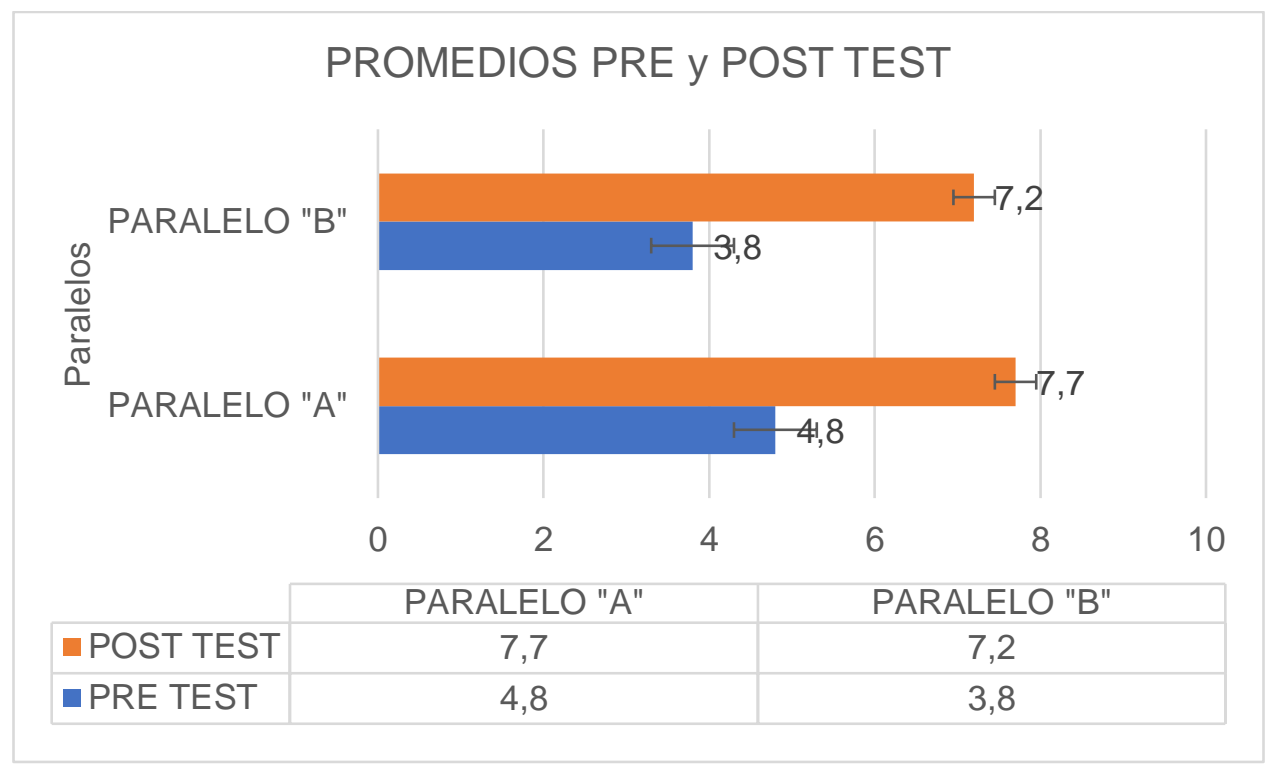

Figura 4

Comparativo de Promedios Pre y Post Test

Fuente: Promedios Pre y Post Test aplicado estudiantes Nivel II paralelo A y B

Autor: Investigadores

Los datos estadísticos que se aprecian en el gráfico 4, demuestran que el promedio general de los 20 estudiantes del Nivel II paralelo "A" era de 4,8 en conocimiento general de vocabulario en inglés y luego de aplicar la plataforma Kahoot durante los tres periodos de estudio, los estudiantes logran obtener un promedio general de 7,7 donde se evidencia claramente el avance en el aprendizaje de nuevo vocabulario a través de la Plataforma educativa Kahoot.

Por otra parte, y de la misma manera los 28 estudiantes pertenecientes al Nivel II paralelo "B" luego de aplicado el Pre test tienen un promedio de 3,8 de conocimientos en vocabulario del idioma inglés, lo cual indicaba un bajo nivel de conocimientos en su vocabulario a la hora de realizar las diferentes actividades, pero luego de haber aplicado las actividades interactivas y simultaneas con la plataforma Kahoot consiguieron un promedio general de 7,2; valor que arrojo al aplicar y tabular los valores en el Post test, lo cual indico a la vez un crecimiento evolutivo de 
nuevo vocabulario en forma ascendente y significativa.

Concluyendo que, la implementación de la plataforma lúdica Kahoot permitió el crecimiento del vocabulario en ingles en los 48 estudiantes del Nivel II de inglés paralelo "A" y "B" de la Carrera de Ingeniería Electrónica en Control y Redes Industriales de la Escuela Superior Politécnica de Chimborazo en la cuidad de Riobamba.

\section{Discusión}

Luego de analizar y describir los diferentes resultados obtenidos de esta investigación, permitieron visualizar que el uso de medios impresos como diccionarios y como también las clases tradicionales no estaban aportando para la adquisición de nuevo vocabulario en inglés, es decir no se estaba aprovechando los medios tecnológicos que nos brinda hoy en día el mundo actual.

Reflexionar y, más, aun, hacer aportaciones y formular propuestas a favor de la mejora de la educación, es siempre una actividad compleja, arriesgada y nunca exenta de dificultades. Y lo es más aún, cuando se realiza desde un terreno innovador y permanentemente cambiante como es el de la tecnología. (Pedró, 2015, pág. 7)

Es de resaltar que el uso de la tecnología en la educación cada día es más útil, razón por la cual la implementación de la plataforma Kahoot permitió el desarrollo del aprendizaje de nuevo vocabulario ajustados a los contenidos del Nivel II de inglés. De esta manera, la plataforma Kahoot como parte del proceso de aprendizaje de nuevo vocabulario permite realizar actividades que motiven en forma constante e interactiva. Además, basado en los datos cuali-cuantitativos (Tablas 1 y 2) se determinó que la implementación de la Plataforma lúdica Kahoot en los dos grupos de estudio si tuvieron cambios considerables en la adquisición de vocabulario en inglés.

La diferencia entre este trabajo de investigación y otros trabajos se centra primordialmente en los resultados obtenidos. Es así, que Nadina Travieso (2017) declara que los resultados son aquellos que permiten enriquecer, modificar o perfeccionar la teoría científica, con el aporte de conocimientos sobre el objeto y los métodos de la investigación. Marcando de esta manera la diferencia de resultados en el aprendizaje de nuevo vocabulario entre el antes y el después de la implementación de la plataforma lúdica Kahoot (Travieso, 2017).

También se presentaron ciertas limitaciones como el miedo y la aceptación de este nuevo método de estudio y el peligro a la no aceptación por parte de los estudiantes y docentes por la falta de familiaridad o desconocimiento de la tecnología. 
Sin embargo, Kahoot, ofreció una experiencia real como herramienta para la enseñanza de vocabulario en inglés donde el docente se convirtió en un facilitador y guía mientras que los estudiantes fueron los protagonistas de su propio aprendizaje.

Finalmente, y luego de considerar los factores que pudieron afectar los resultados de la investigación, tal como: el desconocimiento de la plataforma, temor al uso de la tecnología y la aceptación a nuevas opciones de estudio; los resultados positivos presentados en este estudio sobre la plataforma lúdica Kahoot para el proceso enseñanza aprendizaje de vocabulario en los estudiantes, invita a explorar más a fondo el uso de diversas plataformas, aplicaciones en la educación de hoy en día.

\section{Conclusiones}

En síntesis, desde el análisis de los resultados obtenidos al proponer la implementación de la plataforma Kahoot en la enseñanza de vocabulario en inglés, se puede concluir en tres ideas generales: en primer lugar, que luego de identificar las principales causas del bajo nivel de vocabulario en los estudiantes del Nivel II de inglés de la Carrera de Ingeniería Electrónica en Control y Redes Industriales, se procedió a dejar de lado el uso de diccionarios impresos acompañados de las clases monótonas las mismas que no permitían un buen aprendizaje de vocabulario nuevo en inglés, donde se dio paso al uso de la plataforma Kahoot; como parte del proceso de enseñanza de nuevo vocabulario, siendo de gran utilidad para garantizar los resultados obtenidos.

En segundo lugar, este estudio permitió identificar las principales causas del bajo nivel de vocabulario de los estudiantes de la Carrera de Ingeniería Electrónica en Control y Redes Industriales, las mismas que luego de ser identificadas fueron abolidas en forma sistemática y definitivamente al implementar la plataforma Kahoot dentro del proceso de enseñanza aprendizaje del vocabulario.

Después, de la realización de esta investigación se observó el significado que tiene la tecnología a través de sus diferentes características y beneficios de la plataforma Kahoot en el aprendizaje de vocabulario en inglés. La tecnología se está renovando continuamente, por lo que los docentes debemos estar preparados para estos nuevos retos ya que los estudiantes de ahora están familiarizados con el uso de estas tecnologías desde que nacen.

Finalmente, mencionamos tres actividades las mismas que motivan el aprendizaje de 
ISSN: 2600-5859

vocabulario en inglés con el uso de la plataforma Kahoot, lo cual encierra la premisa de generalizar y sistematizar el uso de esta plataforma en otras aulas de clase, para lo cual nos permitimos recomendar el uso de Kahoot como una necesidad de renovar el que hacer educativo, constatando que contamos con herramientas que al combinarlas pueden ayudar a sacar el máximo provecho en los estudiantes de hoy; quienes nacieron con las nuevas tecnologías y son más activos mientras que los docentes debemos conseguir alumnos interesados en el aprendizaje y con éxito en sus resultados. Kahoot, puede servir como ejemplo de experiencia y herramienta complementaria dentro de las aulas y ayudar a otros docentes en el camino de la innovación educativa.

\section{Referencias bibliográficas}

Espeso, P. (20 de diciembre de 2019). Educación 3.0. Obtenido de https://www.educaciontrespuntocero.com/experiencias/ideas-ensenar-ingles-kahoot/

Gallegos, J. (2015). Kahoot, la mejor manera de aprender jugando. IDEA.

Heraldo, C., \& Jansson, L. (2011). Matices en Lenguas Extranjeras. UNAL, 1-37.

Mansur, M., \& Fadhilawati, D. (2019). Applying Kahoot to Improve the Senior High School Students' Vocabulary Achievement. Voices of English Language Education Society, 164173.

Martínez, G. (2017). Tecnologías y nuevas tendencias de educación: aprender jugando. Opción.

Mendoza, J. (27 de septiembre de 2018). LearnSafe. Obtenido de https://learnsafe.com/usingkahoot-in-the-classroom/

Montaner, S. (2018). The use of KAHOOT in the EFL classroom within the CLIL approach. ResearchGate.

Muñoz, A. (21 de enero de 2014). Wiki Estudiantes. Obtenido de https://www.wikiestudiantes.org/por-que-es-importante-el-vocabulario-en-una-lenguaextranjera/

Pedró, F. (2015). La tecnología y la Transformación de la Educación. Perú: Santillana S.A. 
ISSN: 2600-5859

Vol. 3, N¹, p. 44-62, marzo, 2020

Restrepo, L. (15 de enero de 2016). Investigación Documental. Obtenido de http://aprendeenlinea.udea.edu.co/lms/moodle/file.php/658/Glosario_Invest_Documental final - Lina Rpo.pdf

Rojas, M. (2015). Tipos de Investigación Científica. REDVET, 14. Romero, S. (2016). Didáctica de la lengua inglesa en educación primaria. Soria: Universidad de Valladolid. Susilo, N. (2019). Kahoot Apps in English Language Teaching (ELT) Context: An alternative Learning Strategy. ELSYA, 11-15. Travieso, N. (5 de mayo de 2017). Los resultados científicos en las investigaciones. Obtenido de http://scielo.sld.cu/pdf/san/v21n5/san16215.pdf Universidad Central del Ecuador. (13 de enero de 2015). Metodología de la Investigación. Obtenido de https://es.slideshare.net/juliolara7/capitulo-iii-metodologia-de-investigacin-ejemplo-pdf Villasante, M. (21 de abril de 2017). EFE: Escuela. Obtenido de http:/www.efeescuela.es/noticias/los-robots-tambien-ayudan-aprender-ingles/.

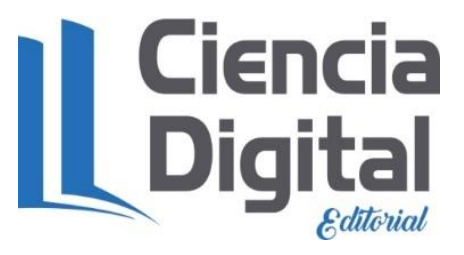


ISSN: 2600-5859

Vol. 3, N¹, p. 44-62, marzo, 2020

\section{PARA CITAR EL ARTÍCULO INDEXADO}

Guano Merino, D. F., Barragán Murillo, R. de los Ángeles, Rodríguez Arellano, N. G., \& Terán Peralta, A. M. (2020). La plataforma lúdica Kahoot en el aprendizaje de vocabulario en inglés. ConcienciaDigital, 3(1.2), 44-62. https://doi.org/10.33262/concienciadigital.v3i1.2.1172

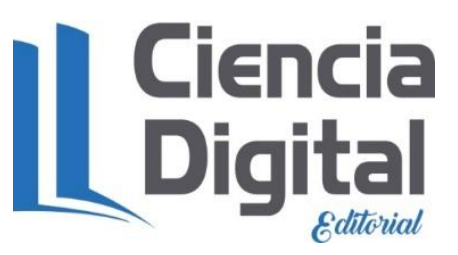

El artículo que se publica es de exclusiva responsabilidad de los autores y no necesariamente reflejan el pensamiento de la Revista Conciencia Digital.

El artículo queda en propiedad de la revista y, por tanto, su publicación parcial y/o total en otro medio tiene que ser autorizado por el director de la Revista Conciencia Digital.

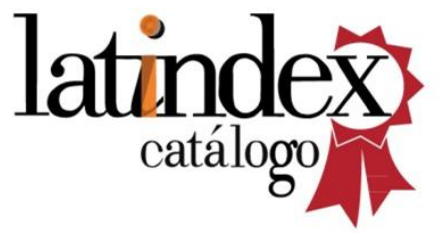

Conciencia

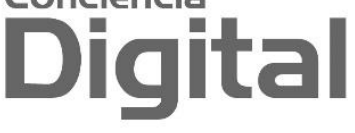

